Revista Perspectivas Online: Biológicas \& Saúde Junho de 2017, Vol.7, n 24 , p. 32-39

ISSN: 2236-8868 (Online)

DOI: $10.25242 / 886872420171163$

\title{
EDUCAÇÃO EM SAÚDE PARA PREVENÇÃO E CONTROLE DO AEDES AEGYPTI
}

\author{
Ana Carolina de Almeida Gomes, Daniela Rios Andrade Lima Tavares', Gabriela Pessanha \\ Beyruth $^{1}$, Thaís Aparecida de Castro Palermo² \& Carolina Magalhães Santos ${ }^{2 *}$
}

\section{RESUMO}

GOMES, A.C.A.; TAVARES, D.R.A.L.; BEYRUTH, G.P.; PALERMO, T.A.C. \& SANTOS, C.M. Educação em saúde para prevenção e controle do aedes aegypti . Perspectivas Online: Biológicas e Saúde, v.7, n.24, p. 32-39, 2017.

Atualmente, a circulação concomitante dos vírus da dengue, zika e chikungunya tem deixado o Brasil em estado de alerta, principalmente, pelo fato de serem transmitidos pelo mesmo vetor, o Aedes aegypti. Neste contexto, o presente estudo tem como objetivo identificar o conhecimento dos moradores de uma comunidade do Campos Goytacazes, município do interior do estado do Rio de Janeiro, Brasil sobre o mosquito, doenças relacionadas e medidas de controle e prevenção e, por meio de ações educativas promover a conscientização dessa população. A metodologia utilizada foi a pesquisa-ação, realizada na comunidade Tamarindo em três etapas. Primeiramente ocorreu a busca ativa dos focos do vetor e aplicação de formulários pré-teste para 33 indivíduos. Posteriormente, foram realizadas ações de educação em saúde por meio de palestras, teatro com as crianças da comunidade e distribuição de panfletos. E, na terceira etapa, realizou-se a aplicação de formulários pós-teste. Ao comparar os resultados dos formulários verificou-se um aumento do percentual de participantes que disseram conhecer o vetor, saber que ele coloca ovos, que se cria, conhecer as doenças relacionadas e a transmissão das mesmas pelo vetor. Neste contexto, constatamos que as ações educativas devem ser trabalhadas continuamente a fim de serem inseridas no cotidiano desses indivíduos na expectativa de assumirem o caráter transformador do processo educativo, favorecendo a prevenção, promoção da saúde e, principalmente, o exercício da construção da cidadania.

Palavra - chave: Enfermagem em saúde pública; educação em saúde; Aedes aegypti 


\begin{abstract}
Currently, the concomitant circulation of dengue virus, zika and chikungunya has left Brazil in a state of alert, mainly because they are transmitted by the same vector, Aedes aegypti. In this context, the present study aims to identify community residents' knowledge about the mosquito, related diseases and control and prevention measures and, through educational actions, promote awareness of the population. The methodology used was the action research in the Tamarindo Community, with active search of the vector foci and application of pre-test forms for 33 individuals. Subsequently, health education actions were carried out through lectures, theater with

community children and distribution of pamphlets. And, finally, application of post-test forms. When comparing the results of the forms there was an increase in the percentage of participants who said they knew the vector, knowing that it places eggs, which is created, know the diseases and the transmission of the same by the vector. In this context, we find that educational actions must be continuously worked in order to be inserted in the daily life of these individuals in the expectation of assuming the transforming character of the educational process, favoring prevention, health promotion and, above all, the exercise of citizenship construction.
\end{abstract}

Keywords: public health nursing; health education; Aedes aegypti

\footnotetext{
${ }^{1}$ Acadêmicos de Enfermagem - ISECENSA - - Rua Salvador Correa, 139, Centro, Campos dos Goytacazes, RJ, CEP: 28035-310, Brazil;

${ }^{2}$ Institutos Superiores de Ensino do CENSA - ISECENSA - Laboratório de Estudos em Saúde Pública - Rua Salvador Correa, 139, Centro, Campos dos Goytacazes, RJ, CEP: 28035-310, Brasil.

(*) e-mail: carolmsantos@yahoo.com.br

Data de chegada: 18/04/2017 Aceito para publicação: 12/06/2017
}

Persp. online: biol. \& saúde, Campos dos Goytacazes, 24 (7), 32-39, 2017

seer.perspectivasonline.com.br 


\section{INTRODUÇÃO}

Atualmente, a circulação concomitante dos vírus da dengue, zika e chikungunya tem deixado o Brasil em estado de alerta, principalmente, pelo fato de serem transmitidos pelo mesmo vetor, o mosquito Aedes aegypti (LIMA-CAMARA, 2016).

A transmissão acontece por intermédio da picada da fêmea do Aedes aegypti e o controle do vetor tem constituído um grande desafio, pois a implementação dos programas não tem alcançado o sucesso esperado. As falhas para o não alcance desse manejo podem estar relacionadas com problemas de infraestrutura das cidades, falta de saneamento básico, os métodos adotados para a contenção do vetor, além da falta de envolvimento da população (ZARA et al., 2016).

De acordo com Silva, Mallmann e Vasconcelos (2015), as estratégias mais comuns na prevenção e combate ao mosquito, são as práxis campanhista/higienista, nas quais são exigidos as populações ações centradas na extinção dos criadouros domésticos, por meio da remoção da água parada de possíveis locais de reprodução do mosquito, tais como pneus, garrafas, e qualquer objeto que possa armazenar água.

Desta forma, a educação em saúde surge como a forma mais eficaz de prevenção contra o mosquito, pois as atividades educativas assumem um valor cada vez maior na elucidação do Aedes aegypti, das doenças transmitidas por ele e sua etiologia, aumentando a participação da população na erradicação dos criadouros (SALES, 2008).

A educação em saúde e a educação ambiental, não acontecem uma sem a outra, e o enfermeiro tem papel fundamental como agente educador em ambos os ensinamentos. A reprodução do Aedes aegypti está relacionada com o ambiente, condições precárias de saneamento básico, higiene, e o próprio conhecimento da população, sobre as medidas de prevenção e controle. O enfermeiro deve usar um modelo de educação em saúde mais dinâmico e que cause maior efeito na população, para que as medidas sejam efetivas e realmente aconteça a prevenção e combate do mosquito (PERES et al. 2015). As abordagens educativas para serem eficazes devem ser criativas e estimular a participação ativa da população (CAREGNATO et al., 2008).

Neste contexto, o presente estudo tem como objetivos identificar o conhecimento dos moradores de uma comunidade do Campos Goytacazes, município do interior do estado do Rio de Janeiro, Brasil, sobre o Aedes aegypti, doenças relacionadas e medidas de controle e prevenção e, por meio de ações educativas promover a conscientização da população, incentivando métodos de prevenção e combate do vetor.

\section{METODOLOGIA}

Trata-se de uma pesquisa-ação, amplamente utilizada no campo da Enfermagem por estar fundamentada na articulação entre teoria e prática estimulando a interação entre os sujeitos de pesquisa e o pesquisador visando a resolução de um problema ou a transformações de uma realidade (CARVALHO E SILVA et al., 2011), realizada na comunidade Tamarindo, Campos dos Goytacazes, estado do Rio de Janeiro, localizada na área central e mais antiga da cidade, na rua Tenente Coronel Cardoso (Formosa) $\mathrm{s} / \mathrm{n}^{\mathrm{o}}$, realizada no período de setembro a novembro de 2016. A amostra foi selecionada por conveniência sendo constituída por 33 indivíduos (um representante de cada domicílio) da comunidade.

A primeira etapa ocorreu em setembro de 2016, durante uma semana, onde realizaram-se as visitas domiciliares com busca ativa dos focos do Aedes aegypti, identificação dos criadouros potenciais nos domicílios e peridomicílios e, aplicação do questionário pré-teste a fim de investigar o nível de conhecimento dos indivíduos sobre o Aedes e doenças relacionadas (dengue, zika e chikungunya) a partir da assinatura do Termo de Consentimento Livre e Esclarecido (TCLE).

A segunda etapa da pesquisa ocorreu em outubro de 2016, também durante uma semana, quando retornamos à comunidade para dar início às ações de educação em saúde por meio de palestra, distribuição

Persp. online: biol. \& saúde, Campos dos Goytacazes, 24 (7), 32-39, 2017

seer.perspectivasonline.com.br 
de panfletos e apresentação de um teatro com as próprias crianças da Comunidade "Chapeuzinho Vermelho e o Aedes Mau". Durante esta semana, realizamos ensaios diários da peça de teatro com as crianças e, também, elaboramos os panfletos e discutimos os principais pontos a serem abordados na palestra. As ações foram realizadas em frente à sede dos Institutos Superiores de Ensino do Centro Educacional Nossa Senhora Auxiliadora (ISECENSA) na comunidade Tamarindo, com os indivíduos que responderam ao questionário pré-teste. A proposta de intervenção educativa visa proporcionar a interação entre todos da comunidade trabalhando de forma-críticareflexiva-prática, em um contexto de educação em saúde, para que a realização seja continuamente posta em prática, sendo incorporada no cotidiano da população.

A terceira etapa da pesquisa ocorreu uma semana após a segunda etapa, no próprio mês de outubro, com o retorno à comunidade para aplicação do questionário pós-teste. E, posteriormente, os dados foram analisados estatisticamente no software SPSS, versão 19.0.

A pesquisa foi desenvolvida de acordo com os princípios éticos da Resolução 466/12 do Conselho Nacional de Saúde tendo sido aprovada pelo Comitê de Ética em Pesquisa com Seres Humanos dos Institutos Superiores de Ensino do CENSA (CEPISECENSA) em 17/06/2016 sob o CAAE de $n^{\circ}$ 56918316.9.0000.5524.

\section{RESULTADOS E DISCUSSÃO}

A primeira etapa deste estudo se consolidou por intermédio da aplicação de formulários, caracterizando a etapa pré-teste. A amostra foi composta por 33 indivíduos. Destes, $69,7 \%$ eram do sexo feminino, com média de idade de 37,9 anos ( $\mathrm{DP}=13,4$ anos), 45,5\% com ensino fundamental incompleto e com renda familiar per capita média de $\mathrm{R} \$ 321,99(\mathrm{DP}=\mathrm{R} \$ 272,33)$, de acordo com a tabela 1.

Tabela 1: Caracterização sociodemográfica de 33 indivíduos da comunidade Tamarindo.

\begin{tabular}{lcc}
\hline \multicolumn{1}{c}{ Variáveis } & N & \% \\
\hline Sexo & 23 & 69,7 \\
$\quad$ Feminino & 10 & 30,3 \\
$\quad$ Masculino & & \\
Idade & 15 & 45,5 \\
$\quad$ De 15 a 35 anos & 10 & 30,3 \\
$\quad$ De 36 a 45 anos & 8 & 24,2 \\
$\quad$ De 46 anos ou mais & & \\
Grau de instrução & 1 & 3,0 \\
$\quad$ Não frequentou a escola & 15 & 45,5 \\
$\quad$ Fundamental incompleto & 8 & 24,2 \\
$\quad$ Médio incompleto & 2 & 6,1 \\
$\quad$ Universitário incompleto & 7 & 21,2 \\
$\quad$ Universitário completo & & \\
Renda Familiar per capita & & 27,3 \\
Até R \$ 220,00 & 9 & 21,2 \\
De R $\$ 221,00$ a R $\$ 440,00$ & 7 & 6,1 \\
De R $\$ 441,00$ ou mais & 2 & 45,5 \\
Não sei./Não quero responder. & 15 & \\
& & \\
\hline
\end{tabular}


Quando questionados sobre o Aedes aegytpi, 66,7\% disseram conhecê-lo, 91,3\% afirmaram que ele é um mosquito, $84,8 \%$ que ele coloca ovos, $84,8 \%$ que possui larvas e $72,7 \%$ que se cria. A maioria (97\%) disse saber sobre a transmissão de doenças pelo vetor, entretanto apenas $27,6 \%$ responderam corretamente quanto à identificação dessas doenças. Apesar da dificuldade apresentada em relacionar o vetor à doença, $73,3 \%$ disseram saber como essas doenças são transmitidas. Quanto à prevalência das doenças transmitidas pelo mosquito, 36,4\% disseram já ter tido casos de dengue e $6,1 \%$ de zika "em casa". Não houveram relatos de casos de chikunguya. Quando questionados sobre o interesse em participar de uma palestra sobre o Aedes aegypti, $73,3 \%$ apresentaram interesse em participar. A maioria $(83,3 \%)$ disse não possuir focos em suas casas. Dos que disseram possuir (17,7\%), 66,7\% apontaram as caixas de água como local de foco. Ao final da entrevista, foi realizado uma avaliação dos possíveis focos do vetor e, em $6,9 \%$ dos domicílios avaliados foram encontrados.

De acordo com Lutinsky et al. (2013) quando nos deparamos com indivíduos alfabetizados julgamos estes capazes de alcançar os objetivos das campanhas promovidas pelo governo para prevenção de criadouros do mosquito e das doenças ocasionadas por ele. No entanto, devemos compreender que as campanhas por si só não obterão êxito se estes indivíduos não estiverem profundamente sensibilizados e engajados na promoção da saúde e bem-estar coletivo. Neste contexto, Caregnato et al. (2008) corroboram este pensamento afirmando que as campanhas de prevenção não produzem transformações no comportamento dos indivíduos. E, Silva et al. (2015) complementam ainda que a educação em saúde apoiada no modelo campanhista assume caráter sazonal não desenvolvendo as mudanças de hábitos $\mathrm{e}$ comportamentos.

Após a etapa do pré-teste, foram realizadas ações de educação em saúde por meio de palestras, teatro com as crianças da Comunidade e distribuição de panfletos (Figs. 1 e 2). Para a etapa posterior, o formulário foi reaplicado a amostra deste estudo. Os resultados pós-testes mostraram um aumento do percentual de participantes que disseram conhecer o Aedes aegypti (81,8\%), que disseram saber que ele coloca ovos $(87,9 \%)$ e que se cria $(90,9 \%)$. Quanto às informações de que se trata de um mosquito, que possui larvas e identificação dos focos em seus domicílios, mantiveram os percentuais do pré-teste. Observou-se um aumento dos percentuais de participantes que disseram saber sobre as doenças e a transmissão das mesmas pelo vetor, conforme apresentado nos gráficos 1 e 2 . Os relatos de casos de dengue, zika e chikunguya mantiveram os mesmos percentuais do pré-teste.

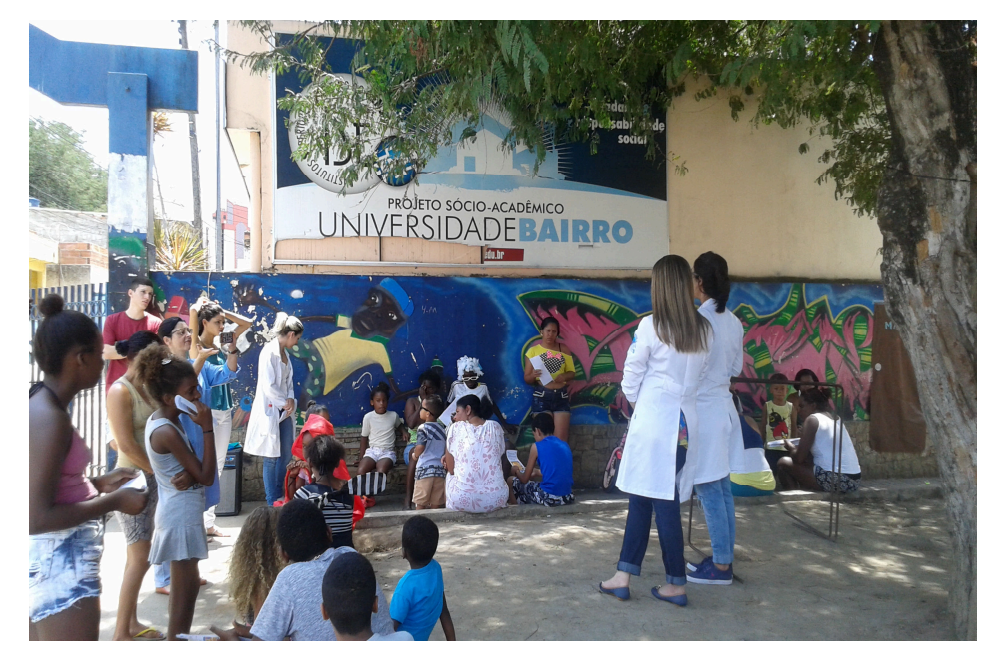

Figura 1: Ações educativas realizadas na Comunidade Tamarindo em Campos dos Goytacazes/RJ: Palestra com distribuição de panfletos sobre o Aedes aegypti e doenças relacionadas.

Persp. online: biol. \& saúde, Campos dos Goytacazes, 24 (7), 32-39, 2017

seer.perspectivasonline.com.br 


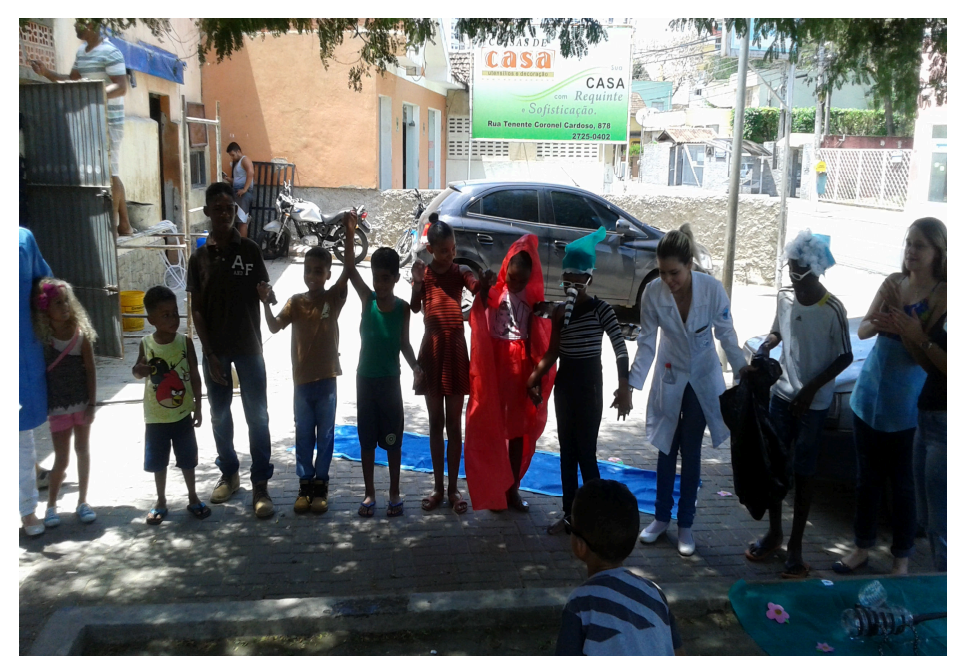

Figura 2: Ações educativas realizadas na Comunidade Tamarindo em Campos dos Goytacazes/RJ: Apresentação da peça de teatro "Chapeuzinho Vermelho e o Aedes mau" com a participação das crianças da Comunidade.

Gráfico 1: Dados Comparativos do pré e pós-teste sobre conhecimentos relacionados ao Aedes

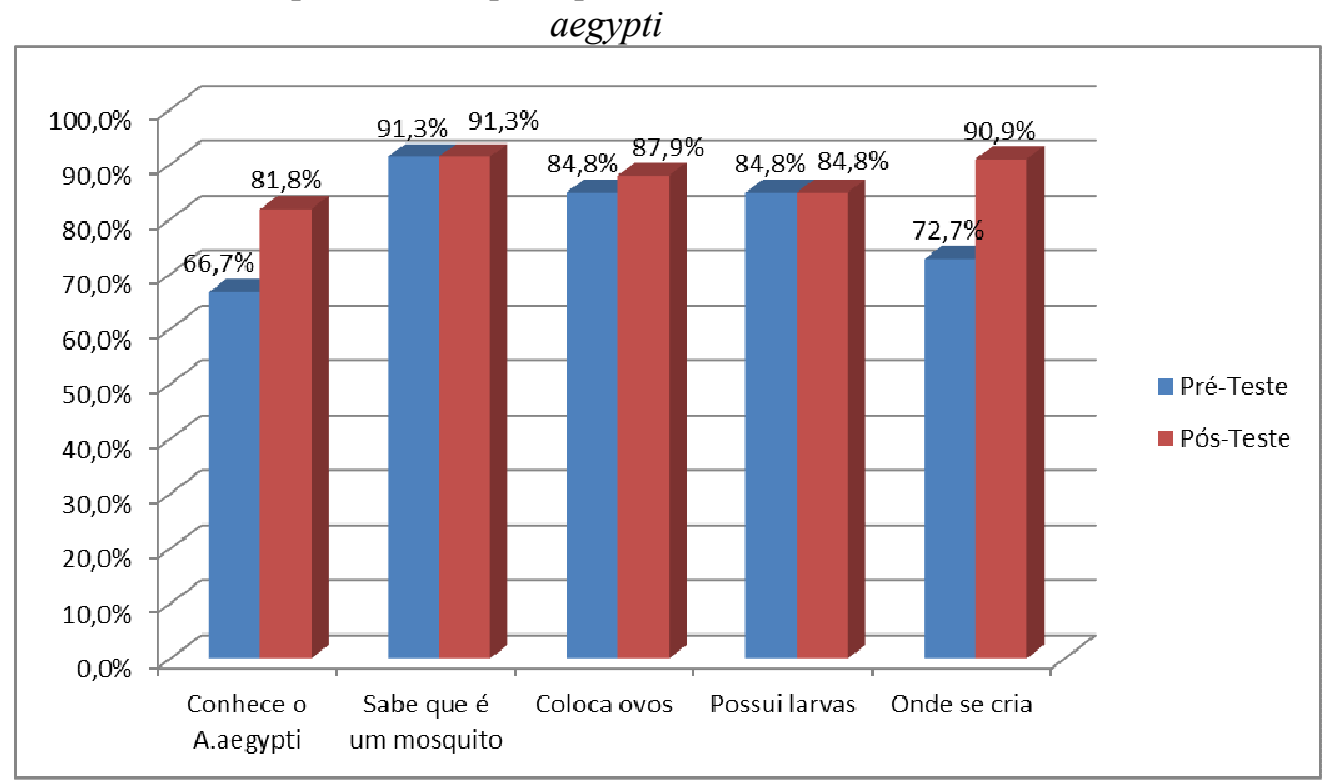


Gráfico 2: Dados Comparativos do pré e pós-teste sobre o Aedes aegypti e doenças relacionadas

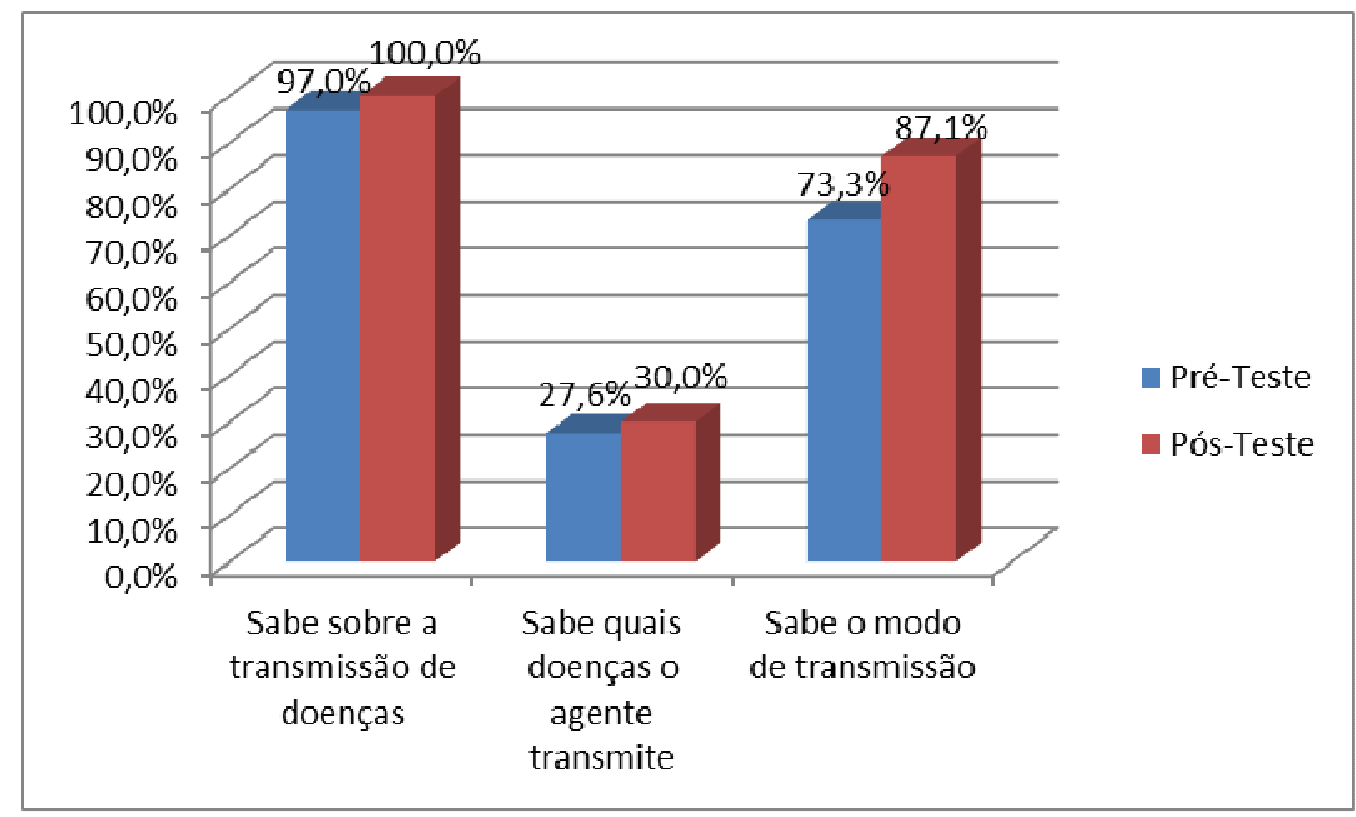

Vale ressaltar, que após a execução da ação educativa, todos apresentaram interesse em participar de palestras para discussões acerca do vetor, evidenciando de forma positiva a importância do desenvolvimento dessas ações entre as comunidades, como forma de conscientizar e sensibilizar para a prevenção e o combate à multiplicação do mesmo. Brassolati \& Andrade (2002) afirmam que as crianças quando verdadeiramente conscientes e envolvidas na problemática a ser trabalhada podem trazer resultados bastante satisfatórios. Oliveira et al. (2012) desenvolveram um estudo onde trabalharam educação e promoção da saúde através da linguagem teatral alcançando resultados positivos levando os indivíduos à refletirem sobre suas ações e a necessidade de mudanças na promoção do bem estar coletivo.

\section{CONCLUSÕES}

Apesar de conhecer o vetor, os hábitos reprodutivos e a transmissão das doenças, a população estudada ainda desconhece quais doenças são transmitidas e apresenta dificuldades na identificação dos focos, impactando no controle e na prevenção das endemias.

Algumas das limitações deste estudo foram o tempo de duração e a fraca adesão dos moradores da Comunidade. Não basta reunir as crianças e seus responsáveis, é preciso que todos estejam envolvidos no processo a fim de não criar lacunas entre a informação e a ação, não podemos permitir a banalização do problema. Além disso, quando trata-se de educação em saúde, a maioria dos autores é taxativo no desenvolvimento da educação continuada a fim de alcançar resultados satisfatórios. Por esta razão, consideramos que nesta ação educativa na Comunidade Tamarindo, a palestra, a distribuição de panfletos e o teatro foram estímulos para despertar a mudança de hábitos nos moradores envolvidos. No entanto, constatamos que as ações educativas devem ser trabalhadas continuamente a fim de serem inseridas no cotidiano desses indivíduos na expectativa de assumirem o caráter transformador do processo educativo, favorecendo a prevenção, promoção da saúde e, principalmente, o exercício da construção da cidadania.

É importante salientar o papel imprescindível do processo educativo mas também é necessário a integração de múltiplas estratégias considerando a demanda e realidade local de cada região para que possamos alcançar uma solução viável na prevenção e controle do Aedes aegypti. 


\section{REFERÊNCIAS}

BRASSOLATTI, R. C. \& ANDRADE, C. F. S. Avaliação de uma intervenção educativa na prevenção da dengue. Ciênc. saúde coletiva [online]. v.7, n.2, 2002. Disponível em: < http://www.scielo.br/pdf/csc/v7n2/10244.pdf >. Acesso em: 30 Jun. 2016.

CAREGNATO, F. F.; FETZER, L. O.; WEBER, M. A. \& GUERRA, T. Educação Ambiental como estratégia de prevenção à dengue no bairro do Arquipélago, Porto Alegre, RS, Brasil. Rev. Bras. de Biociências, Porto Alegre, v. 6, n. 2, abr.jun. 2008. Disponível em: $<$ http://www.ufrgs.br/seerbio/ojs/index.php/rbb/article/view/906/782>. Acesso em: 12 abr. 2016.

CARVALHO E SILVA J.; MORAIS, E. R.; FIGUEIREDO, M. L. F. \& TYRRELL, M. A. R. Pesquisa-ação: concepções e aplicabilidade nos estudos em Enfermagem. Rev Bras Enferm, Brasília, v. 64, n. 3, mai-jun. 2011. Disponível em: <http://www.scielo.br/pdf/reben/v64n3/v64n3a26.pdf>. Acesso em: 10 Abr. 2016.

LIMA-CAMARA, T. N. Arboviroses emergentes e novos desafios para a saúde pública no Brasil. Rev Saúde Pública, São Paulo, v. 50, Epub June 27, 2016. Disponível em: <http://www.scielo.br/pdf/rsp/v50/pt_00348910-rsp-S1518-87872016050006791.pdf>. Acesso em: 20 jul. 2016.

OMS - ORGANIZAÇÃO MUNDIAL DA SAÚDE. Dengue and severe dengue. 2012. Disponível em: $<$ http://www.who.int/mediacentre/factsheets/fs117/en/index.html $>$. Acesso em: 10 abr. 2016.

LUTINSKY, J.A. et al. Infestação pelo mosquito Aedes aegypti (Diptera: Culicidae) na cidade de Chapecó SC. Biotemas, Santa Catarina, v. 26, n. 2, jun. 2013. Disponível em: $<$ https://www.unochapeco.edu.br/static/data/portal/downloads/2724.pdf $>$. Acesso em: 08 Jun. 2016.

PERES, R. et al. Educação ambiental para docentes enfermeiros: percepção e relação com a formação do enfermeiro. Rev. Gaúcha Enferm., Porto Alegre, v. 36, n. spe, 2015. Disponível em: < http://www.scielo.br/scielo.php?script=sci_arttext\&pid=S1983-14472015000500085\&lng=en\&nrm=iso $>$. Acesso em: 30 Jun. 2016.

SALES, F. M. S. Ações de educação em saúde para prevenção e controle da dengue: um estudo em Icaraí, Caucaia, Ceará. Ciênc. saúde coletiva, Rio de Janeiro, v. 13, n. 1, Fev., 2008. Disponível em: $<$ http://www.scielo.br/scielo.php?script=sci_arttext\&pid=S1413-81232008000100022\&lng=en\&nrm=iso $>$. Acesso em: 02 Jun. 2016.

SILVA, I. B.; MALLMANN, D. G.; VASCONCELOS, E. M. R.. Estratégias de combate à dengue através da educação em saúde: uma revisão integrativa. Saúde (Santa Maria), Santa Maria, v. 41, n. 2, jul./dez. 2015. Disponível em: <http://periodicos.ufsm.br/revistasaude/article/view/10955>. Acesso em: 02 Jun. 2016.

SILVA, L.B.; SOARES, S.M.; FERNANDES, M.T.O.; AQUINO, A. 1. Comunicação sazonal sobre a dengue em grupos socioeducativos na atenção primária à saúde. Rev. saúde pública. São Paulo, 2011;v. 45, n. 6. 2011. Disponível em: <http://www.scielo.br/pdf/rsp/2011nahead/2937.pdf $>$. Acesso em: 20 Jul. 2016.

ZARA, A. L. S. A. et al. Estratégias de controle do Aedes aegypti: uma revisão. Epidemiol. Serv. Saude, Brasília, v.25, n. 2, abr-jun. 2016. Disponível em: <http://www.scielo.br/pdf/ress/v25n2/2237-9622-ress-2502-00391.pdf>. Acesso em: 20 Jul. 2016. 